\title{
The Effect of Distinctiveness in Recognizing Average Face: Human Recognition and Eigenface Based Machine Recognition
}

\author{
Naiwala P. CHANDRASIRI ${ }^{\dagger * a)}$, Ryuta SUZUKI ${ }^{\dagger}$, Nobuyuki WATANABE ${ }^{\dagger * *}$, Nonmembers , \\ and Hiroshi YAMADA ${ }^{\dagger}$, Member
}

\begin{abstract}
SUMMARY Face perception and recognition have attracted more attention recently in multidisciplinary fields such as engineering, psychology, neuroscience, etc. with the advances in physical/physiological measurement and data analysis technologies. In this paper, our main interest is building computational models of human face recognition based on psychological experiments. We specially focus on modeling human face recognition characteristics of average face in the dimension of distinctiveness. Psychological experiments were carried out to measure distinctiveness of face images and their results are explained by computer analysis results of the images. Two psychological experiments, 1) Classical experiment of distinctiveness rating and, 2) Novel experiment of recognition of an average face were performed. In the later experiment, we examined on how the average face of two face images was recognized by a human in a similarity test respect to the original images which were utilized for the calculation of the average face. To explain results of the psychological experiments, eigenface spaces were constructed based on Principal Component Analysis (PCA). Significant correlation was found between human and PCA based computer recognition results. Emulation of human recognition of faces is one of the expected applications of this research.

key words: face recognition, distinctiveness rating, average face, principal component analysis (PCA), eigenface
\end{abstract}

\section{Introduction}

Psychologists and computer scientists/engineers have addressed the face perception and recognition problem from different perspectives for decades [1]-[3]. Psychologists have mainly concentrated to reveal cognitive process following perception and to develop information models or to investigate perceptual processing of face patterns [4], [5].

On the other hand, computer scientists/engineers have been interested in finding solutions to the face recognition task for potential applications in such as security and customized services [6], [7].

However, from a broader perspective, there are works that show similarities in characteristics of face recognition in humans and computer based algorithms [4], [8]-[11]. In these works, they consider Principal Component Analysis (PCA) belongs to the right class of image analysis techniques with psychological plausibility.

\footnotetext{
Manuscript received June 9, 2012.

Manuscript revised October 20, 2012.

${ }^{\dagger}$ The author is/was with College of Humanities and Sciences, Nihon University, Tokyo, 156-8550 Japan

*Presently, with Toyota Info Technology center, co.,ltd, Tokyo, 107-0052 Japan

** Presently, with Kanazawa Institute of Technology, Nonoichishi, 921-8501 Japan

a)E-mail: chandxp@gmail.com

DOI: 10.1587/transinf.E96.D.514
}

The main purpose of this study is to perform a novel psychological experiment on recognition of average face and examine their results using computer analysis results based on face spaces for building computer models.

We attempt to investigate some of the results of psychological experiments and computer analysis results of face recognition based on two psychological experiments. 1) Classical experiment of distinctiveness rating of face images and, 2) Novel experiment of recognition of an average face were carried out. PCA based eigenfaces were calculated using stimulus images which were utilized in the psychological experiments. Results of psychological experiments were interpreted by eigenface based computer analysis results. As a result, significant correlation was found between human and PCA based (face spaces) computer recognition.

This paper is organized as follows. In Sect. 2 background of face recognition that is related to this paper is explained briefly. Methods of computer synthesis and analysis of face images that are used in this paper are described in Sect. 3. Two of the psychological experiments, their results and correlation to computer analysis results are given in each of Sect. 4 and Sect. 5. Section 6 is devoted to describing conclusions and future directions of this research.

\section{Background}

There has been a huge amount of research related to face processing in wide variety of fields over the past few decades. Theories have been proposed for explaining how different stages of face processing relate to one another. Two of the main face processing theories are Bruce and Young's model [12] and Burton et al's IAC model [13]. Valentine's multidimensional face space model [14] has been a very important theory on explaining how faces are represented in memory. However there remains a very important question unresolved yet. That is "what information is extracted from faces in order to recognize it?". In answering this question, theories of how faces might be encoded could serve as the 'front end' of the above face processing models. Detailed review of these studies are beyond the scope of this paper. We only discuss findings that are potentially relevant to our work. The interested reader is referred to [15], [16] for detailed information.

Based on Valentine's multidimensional face space model, in recognizing a face by a human, there are three 
known factors that mainly influence the ability of an observer to carry out the task. They are the perceived distinctiveness, orientation and race of the target face [14]. Although, each of these factors had been investigated in isolation based on different theoretical explanations earlier, Valentine proposed a single framework for dealing with all of them. According to his model, individual face is assumed to be encoded as a point in a multidimensional space defined by dimensions that serve to discriminate faces. The main assumption of the framework is that a location in a Euclidean multidimensional space provides an appropriate metaphor for the mental representation of a face.

The process on how faces are represented in the brain, there are mainly two hypotheses, exemplars and face spaces. In the exemplar model, faces are represented by matching to a set of stored exemplars. On the other hand, in the face space model (axis) faces are represented by measuring distances from a standard prototype along a set of different axes [14], [17]. There are some psychophysical evidences for supporting face space model, for example there is a difficulty for exemplar model to explain caricature effect [18], [19]. However, most of the findings can be explained using both models.

In this paper we use face space model in describing results of the experiments. In the literature there are some attempts that have been made to number or label the dimensions of this space [4], [17]. PCA has been influential in building computational models of face recognition that has psychological plausibility. Hancock et al. made comparisons between the responses of a PCA system to a set of test images, and human ratings of facial distinctiveness and similarity for those images. There were significant, although small, correlations between the participants and the PCA system [4], [15]. Their conclusion was that PCA responded much like humans do when trying to remember an image of an unfamiliar face.

The novelty of our research lies in the second psychological experiment where recognition of an average face is carried out and its results are examined by PCA based analysis results. We examined on how the average face of two face images was recognized by a human in a similarity test respect to the original images which were utilized for the calculation of the average face. As a result we found a tendency that humans recognize the average face as more similar to the original image which is placed further away from the origin of a shape-free eigenface space.

\section{Computer Synthesis and Analysis of Face Images}

In this section, we explain how average faces are synthesized for using as stimulus images in psychological experiments and how face images are analyzed based on eigenfaces for interpretation of results of the experiments.

\subsection{Average Face}

We synthesize average face images in terms of the average

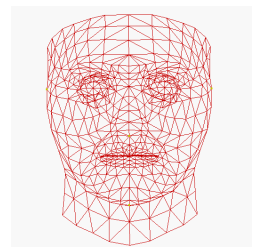

(a) Generic wireframe model

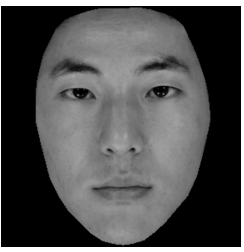

(b) Original image

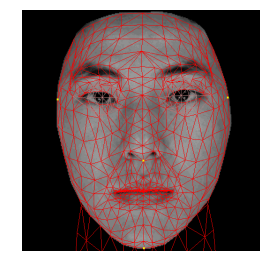

(c) Fitted Wireframe
Fig. 1 Face modeling using FaceFit software.

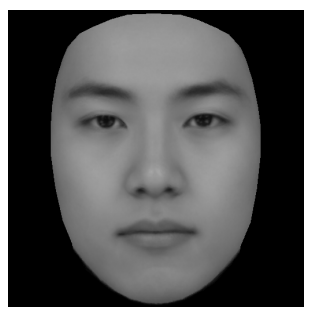

Fig. 2 Average face of twelve face stimuli.

shape and texture [20]. In the process, to avoid blurring of the resulting image, we have to make correspondences between facial feature points such as eyes, eyebrows, mouth, nose etc. of original images and the face model. This can be done by using a wireframe model. First, face modeling is carried out. Figure 1 shows an example of constructing a 3D face model by using FaceFit ${ }^{\dagger}$. A 3D generic wireframe model is shown in Fig. 1 (a). Building a person specific model by fitting the $3 \mathrm{D}$ generic wireframe model on to an original frontal face image (Fig. 1 (b)) is illustrated in Fig. 1 (c). This is done manually by clicking and dragging some feature vertexes of the generic face wireframe model to fit on the original image. We manually do it to make fitting error-free. Some points of the head, face outline, eyes, brows, nose, mouth, and neck (The neck model is eliminated in the calculation of the average face) are utilized as feature points. Other vertexes of the wireframe model are adjusted automatically. Note that the depth values (z) are set to defaults and only $\mathrm{x}, \mathrm{y}$ coordinates are adopted for further calculations. We can save fitted data to a pnt file ${ }^{\dagger \dagger}$. 3D face model with texture is built using texture mapping technique that is based on triangular patches. Average face is constructed by calculating average vertex values of the face wireframe models and then mapping average textures of the corresponding triangular patches on to it. Average face of all of the twelve stimulus images is shown in Fig. 2 .

\subsection{Eigenface}

Principal Component Analysis (PCA) is one of the fre-

${ }^{\dagger}$ Face tools including FaceFit were developed under IPA (face Image Processing system for human like "kansei" Agent) project and, free software download is available under software download service at http://nae-lab.org/project/face/HeikinTool/

${ }^{\dagger \dagger}$ A pnt file contains face shape in terms of $\mathrm{x}, \mathrm{y}, \mathrm{z}$ coordinates of the facial vertexes of the face wireframe model. Once we have a bitmap file and a pnt file of a face image, we can reconstruct the 3D face model 


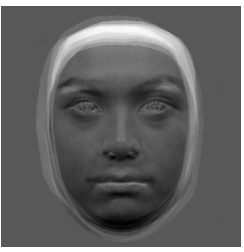

$1^{s t}$

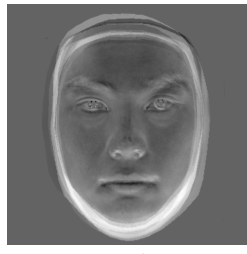

$2^{\text {nd }}$

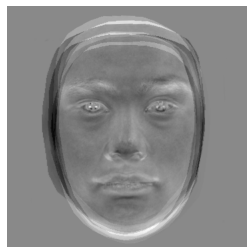

$3^{r d}$

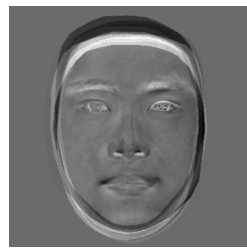

$4^{\text {th }}$

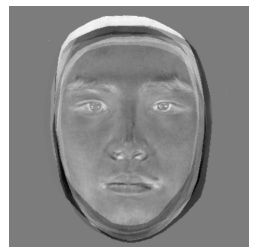

$5^{\text {th }}$

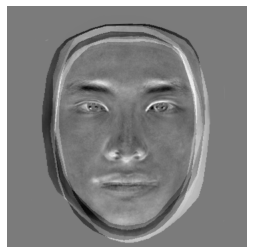

$6^{\text {th }}$

Fig. 3 Full face eigenfaces with the highest eigen values (Affine transformed full faces, reference points: centers of eyes and center of mouth).

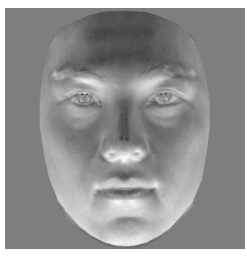

$1^{s t}$

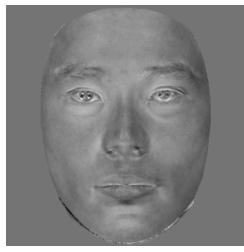

$2^{\text {nd }}$

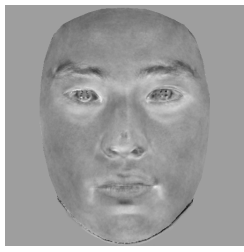

$3^{r d}$

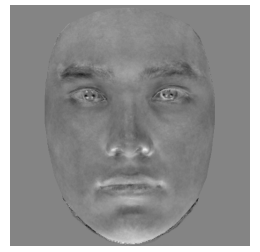

$4^{\text {th }}$

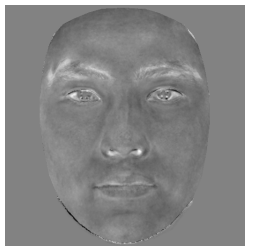

$5^{\text {th }}$

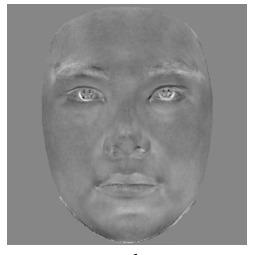

$6^{\text {th }}$

Fig. 4 Shape-free eigenfaces with the highest eigen values (Face shapes are transformed to average shape).

quently used methods for representing face images effectively in low-dimensions. Sirovich and Kirby have proposed an orthogonal basis; eigenpicture for this purpose [21]. Eigenface which was proposed later by Turk and Pentland is one of the well known methods for recognition of face images based on the above orthogonal basis [22], [23]. In the earlier eigenface versions, mainly texture of a face image had been used. We call this full face eigenface method in this paper. For calculating full face eigenface, texture of affine transformed images (three reference points: centers of eyes and center of mouth) are used in this work. Making a rough alignment of pixel values of the same facial parts of different faces is the purpose of the affine transformation. Full face eigenfaces that are calculated from face image data set that is used in the experiment are shown in Fig. 3. These eigenfaces carry information about the variations within the face image data set. Each successive eigenface accounts for progressively less of the variation between faces in the data set [15]. Original faces can be represented by weighted sum of few eigenfaces plus the average face. These weights are the coordinates of the face images on the face space (physical). We use these physical face spaces for calculating computer recognition for full face and shape/shape-free eigenfaces that are described below.

Later, eigenface method was extended by morphing the faces onto an average shape prior to PCA [24]-[26]. This enables the dealing of shape and shape-free texture separately.

In this work, we use $60 \mathrm{key}$ feature points of a face to represent its shape. These points are extracted based on the fitted wireframe model on to the face images. Using these 60 key feature points, shape eigenfaces were calculated.

In the process of calculating full face PCA, we used 3 reference points to make a rough alignment of pixel values. In the shape-free eigenface method we make a better alignment of pixel values of same facial parts of different faces by texture mapping of each face on to the average face shape of the face data set. In the process, the shapes of the faces are lost. Therefore we call them shape-free eigenfaces. Shape-free eigenfaces calculated from our stimuli set (twelve images) are shown in Fig. 4. Artifacts can be seen on the contours of full face eigenfaces in Fig. 3 in comparison to shape-free eigenfaces (Fig. 4). The reasons for the artifacts are variations of contours across original face images due to rough pixel alignment. According to Fig. 3 and Fig. 4, it can be seen that shape-free eigen faces can explain facial variations in terms of the texture to a higher quality.

\section{Experiment 1}

Purpose of this experiment is to perform classical psychological experiment of distinctive rating of face images and then analyze their results based on computer analysis (PCA) of the images that are used in our main experiment (Experiment 2: the novel one). Faces were rated for perceived distinctiveness in this experiment.

\subsection{Participants}

Fifty seven (17 females and 40 males) university students (19 years old in average) made the distinctiveness rating. They were not familiar with any of the people shown to them on the screen. All the participants had normal or correctedto-normal vision.

\subsection{Stimuli}

Twelve face images were randomly selected from a pool of young Japanese male face database ${ }^{\dagger}(512 \times 512$ pixel

\footnotetext{
${ }^{\dagger}$ Twelve images were randomly chosen from facial information database (FIND) [27], [28].
} 
Table 1 Mean distinctiveness rating.

\begin{tabular}{ccccccccccccc}
\hline Face image & $\# \mathrm{a}$ & $\# \mathrm{~b}$ & \# c & \# d & \# e & \# f & $\# \mathrm{~g}$ & $\# \mathrm{~h}$ & $\# \mathrm{i}$ & $\# \mathrm{j}$ & $\# \mathrm{k}$ & $\# \mathrm{l}$ \\
\hline Mean distinctiveness rating & 2.8 & 3.1 & 3.5 & 4.9 & 3.8 & 3.8 & 4.0 & 2.8 & 6.1 & 3.8 & 3.2 & 4.7 \\
\hline
\end{tabular}

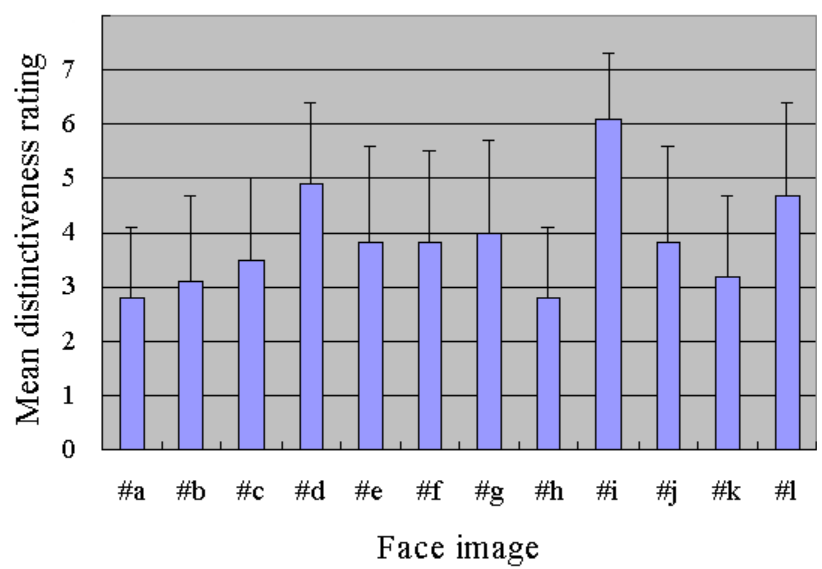

Fig. 5 Mean distinctiveness rating of face images.

Table 2 Correlation coefficients $(r)$ between mean distinctiveness rating of face images and their Euclidean distances from the origin in face space.

\begin{tabular}{cccccccccccc}
\hline Dimensions & $1 \mathrm{D}$ & $2 \mathrm{D}$ & $3 \mathrm{D}$ & $4 \mathrm{D}$ & $5 \mathrm{D}$ & $6 \mathrm{D}$ & $7 \mathrm{D}$ & $8 \mathrm{D}$ & $9 \mathrm{D}$ & $10 \mathrm{D}$ & $11 \mathrm{D}$ \\
\hline Full Face & 0.37 & 0.57 & 0.62 & 0.58 & 0.57 & 0.52 & 0.55 & 0.55 & 0.58 & $\mathbf{0 . 6 3}$ & 0.60 \\
Shape & 0.43 & 0.43 & 0.48 & 0.48 & 0.48 & 0.48 & 0.49 & $\mathbf{0 . 5 0}$ & 0.50 & 0.49 & 0.49 \\
Shape free texture & 0.36 & 0.39 & 0.33 & 0.43 & 0.39 & 0.42 & 0.43 & 0.45 & 0.45 & $\mathbf{0 . 4 6}$ & 0.45 \\
\hline \multicolumn{7}{c}{ Note: $r=0.58$ is significant at 0.05 , two-tailed test. }
\end{tabular}

grayscale images, 256 gray levels). All were images in full face pose with a neutral expression. Moles and facial hair on the original images were removed manually. Face area was extracted using the wireframe model eliminating hair, neck and the background. Background is set to black color. Since user responses to a displayed image on a computer screen may differ with face image size and brightness, height of a face image was kept to $7 \mathrm{cms}$ on the screen and the brightness of the image on the computer screen was set to $20 \mathrm{~cd} / \mathrm{m}^{2}$ (Note that the measured brightness of a face image on the screen and its perceived brightness on the paper may differ).

\subsection{Procedure}

In a class room, face images were displayed on computer screens (TEKGEMS, AS-1503SG, 15 inch, TFT) of individual students. The procedure for rating the faces was the same as described by Valentine \& Bruce [29]. Participants were asked to rate each face on a 1-7 scale. They were instructed to imagine that they had to meet each person for the first time at a railway station and to rate each face for how easy it would be to spot in a crowd. A face that was very distinctive and so would be easy to spot in a crowd should be rated 7. On the other hand a typical face that would be difficult to identify in a crowd should be rated 1 . To make rating, images were selected by a student from a list on a webpage and, each student was provided with a unique random order of a face list. participants were allowed to select and rate face images at their own pace. However, instructions were given them to rate the images as quickly as possible.

\subsection{Results and Discussion}

Mean distinctiveness rating of faces are shown in Table 1 and Fig. 5. Mean distinctiveness rating varies from 2.8 to 6.1 and, overall average is 3.9.

In the conventional face space model, it is assumed that storing representations of faces in memory involves the abstraction of a face norm [14], [17]. Each individual face is stored in memory according to its deviation from a single general face norm which is located at the origin of the face space. Based on this hypothesis, we used the Euclidean distances from the origin in eigenface space as a measure in calculating computer recognition. Note that as an approximation of the face norm, the average face of twelve face stimuli (Fig. 2) is used in this work.

The rated mean distinctiveness of face images and the Euclidean distances from a prototype face (average face) in PCA based face spaces have high correlations (Table 2). Significant correlation is found in full face eigenface space $(r=0.63)$.

In face recognition, these results support the Valentine's framework of face space model whose axes are defined by eigenfaces. 


\section{Experiment 2}

Purpose of this experiment is to perform a psychological experiment of recognition of an average face and analyze their results based on computer analysis (PCA) results of the images for building computational models. The novelty of our research lies in this psychological experiment where recognition of an average face is carried out and its results are examined by PCA based analysis results. We conducted a psychological experiment on how average face of two face images was recognized by a human in a similarity test respect to the original images which were utilized for the calculation of the average face. PCA based algorithms were utilized to investigate the results of the psychological experiments and computer analysis results [30].

We can calculate the average face image $\mathrm{C}$, from two original face images, say $\mathrm{A}$ and $\mathrm{B}$, using image processing techniques in terms of the average shape and texture as described in Sect. 3.1.

However, there is a very basic question one can arise regarding the average face that is calculated based on average shape and average texture values. Is the average face $\mathrm{C}$ recognized as the average of $\mathrm{A}$ and $\mathrm{B}$ by a human? This basic question is a multidisciplinary one. It may have applications in engineering, psychology, neuroscience etc. If the answer to the above question is negative, more questions may arise. Can we explain human response results of the average face? Can we predict the human recognition of $\mathrm{C}$ using some image analysis methods of A and B?

\subsection{Participants}

Thirty Japanese university students (14 females and 16 males) participated in the experiment voluntarily. Their average age was 21.9 years and they did not overlap with the participants of the experiment 1 . Also, they were not familiar with images of any of the people shown to them on the screen. All the participants had normal or corrected-tonormal vision. One participant took part in the experiment at a time.

\subsection{Stimuli}

The main stimuli were twelve face images that were used in the experiment 1 . In addition to that, sixty-six $(12 \times 11 / 2=$ 66) average faces were synthesized based on the procedure given in the Sect. 3.1 for each of the image pairs of twelve images. As moles and facial hair on the original images had been removed, the resulting average face did not contain any obtrusive objects.

\subsection{Procedure}

As shown in the Fig. 6, three images were displayed on the middle of a computer display. The average face image was shown on the upper part and, the two original images that

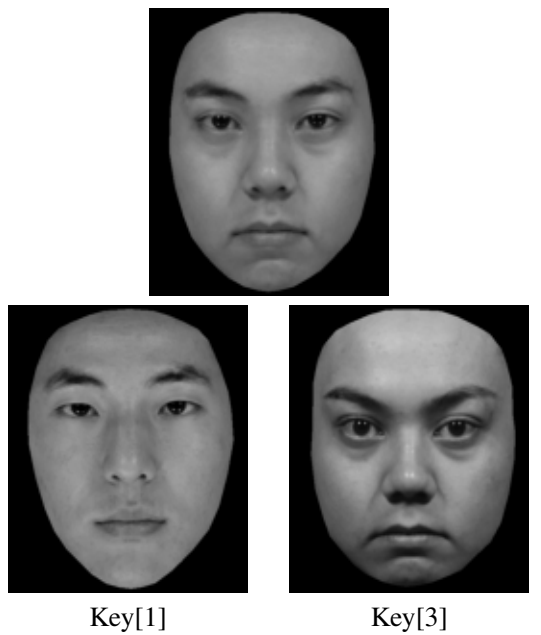

Fig. 6 Average face (on the upper part) was compared with two of the face images that were utilized for its calculation (on the lower part) for similarity as a whole.

were used for the calculation were shown on the lower part of the display. To avoid position effect, positions of the stimulus images in the lower part of the screen were counterbalanced in the experiments. One set of the experiment consisted of 132 trials $(66 \times 2)$ and, stimuli were presented in random order. Participants were seated on a chair $90 \mathrm{~cm}$ away from the display (Dell E152FPC, 15 inch, TFT). They were instructed to compare the average face with two of its original images carefully and select which was more similar to the average face by pressing a key out of two marked keys on a keypad (ELECOM, TK-UYLG). They did not know how we selected or processed the images. In the judgements, They were asked to focus on the 'face as a whole' not on specific facial parts. They were also asked to judge as quickly as possible.

\subsection{Results and Discussion}

First, Kendall's coefficient of concordance was computed for all participants' responses $\left(W=0.467, \chi^{2}(11)=154.22\right.$, $p<.001)$ and, there was a significant agreement among participants. Also, average responses of the participants were distributed nonuniformly across original images (see the row of the average participant responses in the Table 4). In 35 cases among 66 average faces used in the experiment, significant number of responses have been acquired by one of the original images $\left(\chi^{2}(1)=6.63, p=.01\right)$. If there is no bias towards original face images $(\# a \sim \# l)$ which are utilized for calculating average face images, it's expected that there is no significant differences of participant's responses to original face images. As the responses are nonuniformlydistributed across original face images with a significant agreement among participants, it is suggested that there is a bias of recognizing an average face image.

In the Experiment 2 we use the correlation between the average responses and the original face images' distances from the origin of the face space for evaluating the results. 
Table 3 Correlation coefficients $(r)$ between average participant responses and Euclidean distances from the origin in face space.

\begin{tabular}{cccccccccccc}
\hline Dimensions & $1 \mathrm{D}$ & $2 \mathrm{D}$ & $3 \mathrm{D}$ & $4 \mathrm{D}$ & $5 \mathrm{D}$ & $6 \mathrm{D}$ & $7 \mathrm{D}$ & $8 \mathrm{D}$ & $9 \mathrm{D}$ & $10 \mathrm{D}$ & $11 \mathrm{D}$ \\
\hline Full face & 0.51 & 0.50 & 0.47 & 0.43 & 0.35 & 0.33 & 0.39 & 0.37 & 0.44 & 0.42 & 0.44 \\
Shape & 0.35 & 0.20 & 0.22 & 0.18 & 0.20 & 0.20 & 0.20 & 0.20 & 0.20 & 0.20 & 0.20 \\
Shape free texture & 0.85 & 0.87 & 0.87 & 0.89 & 0.91 & 0.92 & 0.93 & 0.94 & 0.93 & 0.92 & 0.91 \\
\hline \multicolumn{7}{c}{ Note: $r=0.58$ is significant at 0.05 , two-tailed test. }
\end{tabular}

Table 4 Average participant responses and Euclidean distance $\left(\times 10^{3}(8 \mathrm{D})\right)$ from the origin on the shape-free eigenface space.

\begin{tabular}{ccccccccccccc}
\hline Face image & $\# \mathrm{a}$ & $\# \mathrm{~b}$ & $\# \mathrm{c}$ & $\# \mathrm{~d}$ & $\# \mathrm{e}$ & $\# \mathrm{f}$ & $\# \mathrm{~g}$ & $\# \mathrm{~h}$ & $\# \mathrm{i}$ & $\# \mathrm{j}$ & $\# \mathrm{k}$ & $\# \mathrm{l}$ \\
\hline Average participant responses & 7.5 & 11.7 & $\mathbf{1 5 . 5}$ & 10.8 & 10.3 & 6.4 & 11.5 & 11.6 & $\mathbf{1 6 . 9}$ & 12.9 & 8.6 & 8.3 \\
Euclidean distance from origin & 4.2 & 6.6 & $\mathbf{9 . 0}$ & 4.8 & 5.9 & 3.8 & 7.5 & 6.9 & $\mathbf{1 1 . 8}$ & 6.5 & 4.0 & 4.2 \\
\hline
\end{tabular}

We use distances from the origin of face space in this paper based on Valentine's and Johnston's et al. work [14], [17]. Their assumption is that distinctive faces are stored in the outer side of the space while typical ones tend to be located towards its center. In this paper we assume that the perceived distinctiveness of a face can be described by its distance from the origin (overall average face or a prototype face) of the space and average face recognition results can be explained based on them. By doing so, we also can analyze all the results of experiment 2 at once rather than carrying out just pairwise comparisons.

For comparison of human and computer recognition, full face, shape-free and shape eigenface spaces are constructed. The highest correlation between average participant responses and Euclidean distances of the original face images from the origin is found in the shape-free eigen space (Table 3 ). In the Table 3, correlation coefficients $(r)$ between average participant responses and Euclidean distances from the origin are shown for 1 to 11 dimensions in fullface, shape and shape-free eigenface space. Significant correlation is found even using a single dimension. The maximum correlation of $0.94(p<.001)$ is seen using 8 dimensions. See Fig. 8 for plotted data of average participant responses vs. distances from the origin on the shapefree eigenface space using 8 dimensions.

In shape and full face eigenface space, correlations of 0.35 and 0.51 in maximum $(r=0.58$ is significant at 0.05 , two-tailed test) can be seen between average participant responses and Euclidean distances from the origin.

In this experiment, average face image of two face images is compared with the original face images for similarity. Thus this experiment focuses on recognizing an average face (combination of average shape and average (shape-free) texture). As experiment results, unsurprisingly, any significant bias is not evident in recognizing the face shape of the average face image. However, there is a noticeable bias in the face space of shape-free texture. The striking finding here is that the correlation between results of the face perception experiment and PCA based face analysis results of the average shape/average shape-free texture have different characteristics. Although, both shape and texture plays a key-role in recognizing a face, as per average face, when comparing to their original face images, presentation on shape-free eigen space and human perception results have the highest and significant correlation.

As it is explained in the results and discussion of the experiment 1, our results supported the Valentine's frame work of face space model whose axes are defined by eigenfaces. In this face recognition model, each individual face is stored in memory according to its deviation from a single general face norm which is located at the origin of the face space. Based on this assumption we used the Euclidean distances from the origin in eigenface space as a measure in calculating computer recognition. Typical faces are generally located near the center of the space while distinctive faces tend to be present in the outer region in a face space [17]. According to our experiment, farther away an original face is situated from the origin, the more number of responses it tend to acquire. According to the Table 4, \#c and \#i images have received higher number of responses in average from the participants and, they are placed rather away from the origin (See also Fig. 7). On the other hand $\# a, \# f, \# k$ and $\# l$ images have received lower number of responses and, they are situated nearer in the distance to the origin. These data are shown in different fonts in Table 4.

The face images used in this experiment are randomly chosen images from the face image database FIND. The database consists of 150 face images and it has a wide variety of Japanese face images. Although the number of face images that were used for psychological experiments were twelve, we believe that the selected images reflect the variations of the original face database.

In the literature there are reports that shape-free texture has some interesting properties [4]. In memory experiments, analysis based upon PCA of shape-free faces has given the highest predictions of false positives; those faces easy to reject as being unseen. In face recognition experiments shapefree representation is tested to be efficient in terms of bit rate and correct hits, by comparing to shape-free plus shape data [31]. As pointed out in above works, the efficiency of shape-free representation does not mean that shape is unimportant. Specially, in our experiment of face recognition by humans, unsurprisingly there was no significant bias could be seen in terms of shape of the face image $C$ towards $A$ or $\mathrm{B}$ ( $\mathrm{C}$ has the average shape and texture of face images $\mathrm{A}$ and $\mathrm{B})$. In other words, there is no evidence that average 


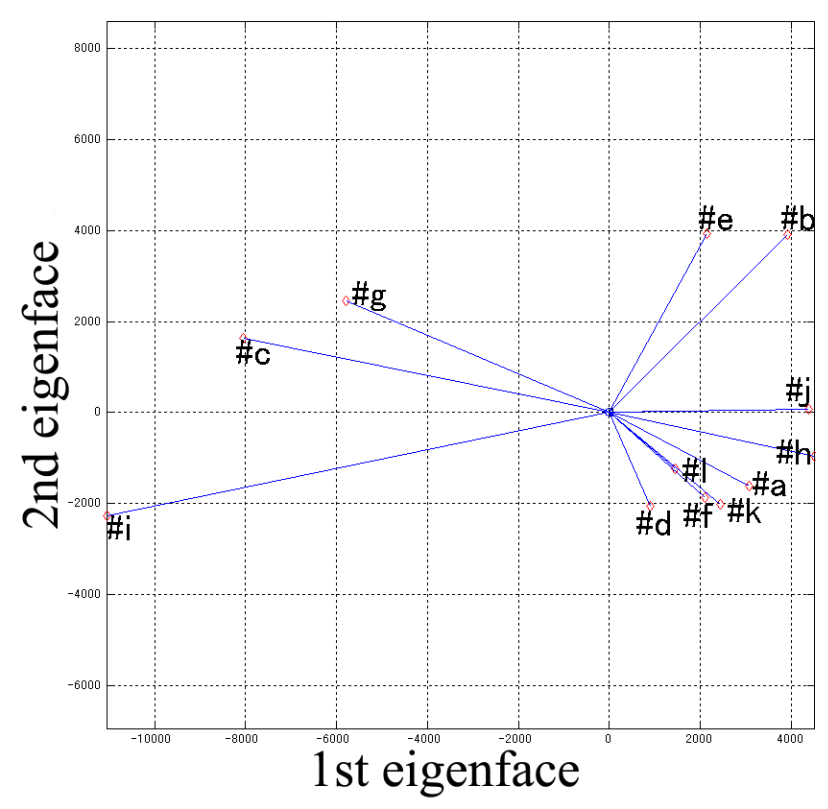

Fig. 7 A face space: Twelve face stimuli on shape-free eigenface space.

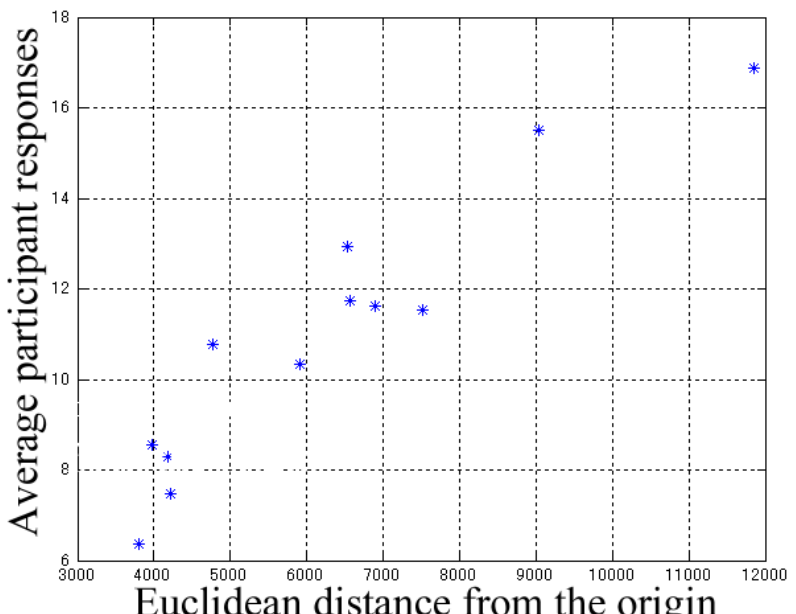

Fig. 8 Correlation between average participant responses and distances from the origin on the shapefree eigenface space (8D).

shape has not been recognized as the average unsurprisingly. There is a bias in recognizing $\mathrm{C}$ in a similarity test to $\mathrm{A}$ and $\mathrm{B}$ and that can be explained based on shape-free face space.

We also calculated the correlation coefficients between results of the two psychological experiments. Medium level correlation, $r=0.41$ ( $r=0.58$ is significant at 0.05 , twotailed test) was found between mean distinctiveness rating of face images in experiment 1 and average participant responses in experiment 2 .

The nature of the two experiments differs in the following perspectives. In the first experiment of distinctive rating, the participants have no other images to compare at their hand and they try to figure out how easy the given image to be spotted in a crowd in an imaginary scenario. Therefore, depending of the participant and the context of their mind experiment, different aspects may affect in making a decision. On the other hand, the second experiment is carried out in more controlled conditions as the participant can compare the given average image with two of their original images. Further research will be conducted in future to investigate these interesting aspects and correlation between the experiments.

\section{Conclusions and Future Directions}

Our main interest is building computational models of human face recognition based on psychological experiments. In this paper, we specially focused on modeling human face recognition characteristics of average face in the dimension of distinctiveness. We conducted one classical and one novel psychological experiment of face recognition, and then explored their correlations with PCA based computer 
recognition.

In the first experiment, significant correlation was found between mean distinctiveness rating of face images and their Euclidean distances from the origin in a PCA based face space (for full faces). This result supported the Valentine's face space model in which eigenfaces served as concrete dimensions in discriminating individual faces.

As a result of the second experiment, we found that there was a bias in recognition of an average face. This bias can be explained using the same framework of Valentine's face space model. When an average face is synthesized from two original face images, there is a tendency that humans recognize it as more similar to the original face image which is placed further away from the origin of a shape-free eigenface space.

According to the results of the experiments, significant correlations were found between human and PCA based computer recognition.

Understanding human characteristics/biases in recognizing facial images and building computational models that can deal with them is an important task. Specially, they would be helpful for collaborative face image processing tasks between human and computer. When human makes a decision in a face recognition task, it can be biased due to certain aspects of human face recognition characteristics. Therefore, it is desirable for computer to support in these tasks by modeling/emulating these characteristics and provide the human with scientific evidence to make the right decision. One of the areas that can have applications of this research in future would be law enforcement and security. An example of such a task is a witness and computer system collaborates to find a suspect's face image.

Carrying a psychological experiments using a larger face image database that consists of wide variety of face images and analyzing their results using a computer based image processing method is remained as our future work. Other future works include synthesizing average faces that are recognized by humans as averages.

\section{Acknowledgements}

The first author, N.P. Chandrasiri was a research fellow of the Japan Society for the Promotion of Science (JSPS), and he also was a guest researcher at the Intelligent Modelling Laboratory (IML), the University of Tokyo at the time when this research was conducted.

Special thanks go to Hiroshi Harashima, Prof. of Emeritus, the University of Tokyo, for his constructive comments and Tatsuo Yotsukura of Advanced Telecommunications Research Institute International (ATR), Japan for his technical support.

\section{References}

[1] V. Bruce and A. Young, In the eye of the beholder; The science of face perception, Oxford University Press, 1998.

[2] C. Curio, H. Blthoff, and M. Giese, Dynamic Faces; Insights from experiments and computation, The MIT Press, 2011.
[3] M. Wenger and J. Townsend, eds., Computational, geometric, and process perspectives on facial cognition: Contexts and challenges (Scientific Psychology Series), Lawrence Erlbaum Associates 2001.

[4] P.J.B. Hancock, A. Burton, and V. Bruce, "Face processing: Human perception and principal components analysis," Memory \& Cognition, vol.24, pp.26-40, 1996.

[5] P. Sinha, B.B. Balas, Y. Ostrovsky, and R. Russell, "Face recognition by humans: Nineteen results all computer vision researchers should know about," Proc. IEEE, vol.94, no.11, pp.1948-1962, 2006.

[6] M. Kaneko and O. Hasegawa, "Processing of face images and its applications," IEICE Trans Inf. \& Syst., vol.E82-D, no.3, pp.589600, March 1999.

[7] P. Phillips, H. Wechsler, J. Huang, and P. Rauss, "The feret database and evaluation procedure for face recognition algorithm," Image and Vision Computing, vol.16, no.3, pp.295-306, 1998.

[8] A. O'Toole, K. Deffenbacher, D. Valentin, and H. Abdi, "Structural aspects of face recognition and the other race effect," Memory \& Cognition, no.22, pp.208-224, 1994.

[9] A.J. Calder, A.M. Burton, P. Miller, A.W. Young, and S. Akamatsu, "A principal component analysis of facial expressions," Vision Research, vol.41, no.9, pp.1179-1208, 2001.

[10] P.J.B. Hancock, A.M. Burton, and V. Bruce, "A comparison of two computer-based face identification systems with human perceptions of faces," Vision Research, vol.38, no.15-16, pp.2277-2288, 1998.

[11] A. Burton, R. Jenkins, P. Hancock, and D. White, "Robust representation for face recognition: The power of averages," Cognitive Psychology, vol.51, pp.256-284, 2005.

[12] V. Bruce and A. Young, "Understanding face recognition," British Journal of Psychology, vol.77, pp.305-327, 1986.

[13] A. Burton, V. Bruce, and R. Johnston, "Understanding face recognition with an interactive activation model," British Journal of Psychology, vol.81, pp.361-380, 1990.

[14] T. Valentine, "A unified account of the effects of distinctiveness, inversion and race of face recognition," Q.J.Exp.Psychol, vol.43A, no.2, pp.161-204, 1991.

[15] G. Hole and V. Bourne, Face processing: Psychological, neuropsychological, and applied perspectives, Oxford University Press, 2010.

[16] W. Zhao, R. Chellappa, P. Phillips, and A. Rosenfeld, "Face recognition: A literature survey," ACM Computing Surveys, vol.35, no.4, pp.399-458, 2003.

[17] R.A. Johnston, A.B. Milne, and C. Williams, "Do distinctive faces come from outer space? an investigation of the status of a multidimensional face-space," Visual Cognition, vol.4, no.1, pp.59-67, 1997.

[18] G. Rhodes, S. Brennan, and S. Carey, "Identification and ratings of caricatures: Implications for mental representations of faces," Cognitive Psychology, vol.19, no.4, pp.473-497, 1987.

[19] D.Y. Tsao and W.A. Freiwald, "What's so special about the average face?," Trends in Cognitive Sciences, vol.10, no.9, pp.391-393, 2006.

[20] A. Nagata, M. Kaneko, and H. Harashima, "Analysis of facial impressions using average faces," Electronics and Communications in Japan, vol.81, no.11, pp.29-35, 1999.

[21] L. Sirovich and M. Kirby, "Low-dimensional-procedure of characterization of human faces," J. Optical Society of America, vol.4, no.3, pp.519-524, 1987.

[22] M. Turk and A.P. Pentland, "Eigenfaces for recognition," J. Cognitive Neuroscience, vol.3, no.1, pp.77-96, 1991.

[23] M. Turk, "A random walk through eigenface," IEICE Trans. Inf. \& Syst., vol.E84-D, no.12, pp.295-306, Dec. 2001.

[24] H. Harashima, T. Okazaki, C. Choi, and T. Takebe, "Principalcomponent analysis of facial images and its applications," IEICE Technical Report, HC90-28, 1991.

[25] I. Craw and P. Cameron, "Parameterising images for recognition and reconstruction," British Machine Vision Conference, pp.367-370, 1991.

[26] I. Craw, N. Costen, T. Kato, G. Robertson, and S. Akamatsu, "Auto- 
matic face recognition: Combining configuration and texture," Proc. Intl. Workshop on Automatic Face and Gesture Recognition, pp.53$58,1995$.

[27] N. Watanabe, R. Suzuki, H. Yoshida, D. Tsuzuki, A. Bamba, N.P. Chandrasiri, G. Tokita, M. Wada, S. Morishima, and H. Yamada, "Facial information norm database (FIND): Constructing a database of Japanese facial images," Jpn. J. Research Emotions, vol.14, no.1, pp.39-53, 2007 (in Japanese).

[28] T. Yamaguchi, N. Watanabe, R. Suzuki, Y. Amano, M. Mizunuma, and H. Yamada, "A fundamental examination of constructing the facial information database," IEICE Technical Report, HCS2002-51, 2003 (in Japanese).

[29] T. Valentine and V. Bruce, "The effect of distinctiveness in recognizing and classifying faces," Perception, vol.15, no.5, pp.525-535, 1986.

[30] N.P. Chandrasiri, R. Suzuki, N. Watanabe, H. Yamada, and H. Harashima, "Is average face recognized as the average," IEEE International Symposium on Signal Processing and Its Applications (ISSPA), 2007.

[31] A.M. Burton, V. Bruce, and P.J.B. Hancock, "From pixel to people: A model of familiar face recognition," Cognitive Science, vol.23, no.1, pp.1-31, 1999.

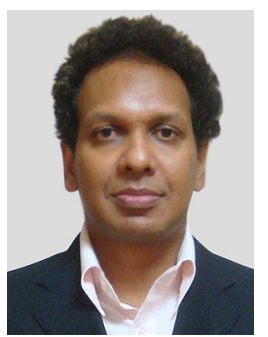

Naiwala P. Chandrasiri received his B.E. and M.E. degrees from the Toyohashi University of Technology in 1996 and 1998 respectively. $\mathrm{He}$ also received his Ph.D. degree in information and communication engineering from the University of Tokyo in 2001. He was a research associate at the University of Tokyo, a guest researcher at the Intelligent Modelling Laboratory (IML), the University of Tokyo, a research fellow of the Japan Society for the Promotion of Science (JSPS) at the Nihon university Japan. Currently he is a researcher at Toyota Info Technology center, Japan. His research interests include computer vision, machine learning, data mining, human machine interface and human communication engineering. He received a best paper award from World Multi-Conference on Systemics, Cybernetics and Informatics, USA in 2001, Niwatakayanagi award from The Institute of Image Information and Television Engineers in 2007, and Best Conference Paper (Development and Implementation) from IEEE International Conference on Cyber Technology in Automation, Control, and Intelligent Systems, Thailand in 2012.

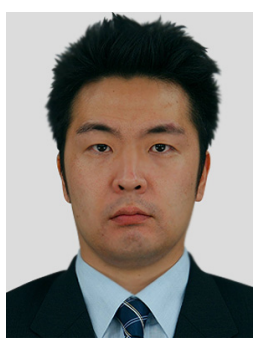

Ryuta Suzuki received the B.A., M.A., and Ph.D. in Psychology from Nihon University, Tokyo, Japan, in 1998, 2000, and 2005, respectively. He is a research fellow at the Institute of Information Sciences, College of Humanities and Sciences, Nihon University. He doubled as research fellow of Japan Society for the Promotion of Science from 2000 to 2003. His research interest has involved human communication and emotion, especially approaches of the recognition of facial expressions of emotion through Cognitive Psychology and Psychophysiology. He is a member of Japanese Psychological Association, The Japanese Psychonomic Society, The Japanese Society for Cognitive Psychology, and The Japanese Society for Physiological Psychology and Psychophysiology.

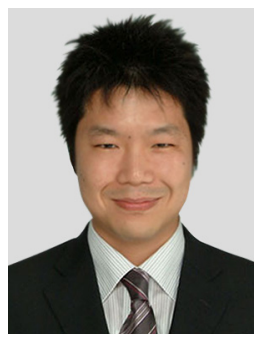

Nobuyuki Watanabe received the B.A., M.A., and Ph.D. in Psychology from Nihon University, Tokyo, Japan, in 2001, 2003, and 2006 respectively. He is an assistant professor at the Department of Psychological Informatics, College of Informatics and Human Communication, Kanazawa Institute of Technology, Ishikawa, Japan. His major field of interest is cognitive psychology on communication, especially facial expressions of emotion. $\mathrm{He}$ is a member of Japanese Psychological Association, Japanese Academy of Facial Studies, The Japanese Psychonomic Society, The Japanese Society for Cognitive Psychology, Human Interface Society, The Japan Society for Research on Emotions, and Hokuriku Psychological Society of Japan.

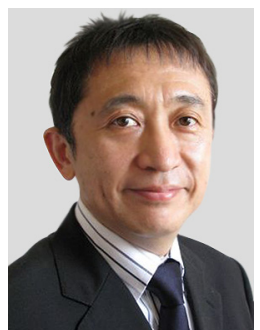

Hiroshi Yamada received the B.A., M.A. and Ph.D. in Psychology from Nihon University, Tokyo, Japan, in 1981, 1983 and 1997, respectively. He is a professor at the Department of Psychology, College of Humanities and Sciences, Nihon University. He was an invited researcher, ATR Human Information Processing Research Laboratory in Kyoto from 1999 to 2000, an invited scientist, National Space Development Agency of Japan in Tsukuba from 2002 to 2003, a visiting researcher, NASA Ames Research Center in California, USA from 2003 to 2004. He was a chairman of Human Communication Group of the Institute of Electronics, Information and Communication Engineers (IEICE) in 2011. His research interests include the recognition of facial expression, the face perception, and human communication. He received the Research Encouraging Award and the Outstanding Research Paper Award in 1994 and 2002 respectively from Japanese Psychological Association. He is a member of Japanese Psychological Association, Japanese Psychonomic Society, and Japan Academy of Facial Studies. 